\title{
Assessment of the Gastrointestinal Fate of Bacterial Cellulose and Its Toxicological Effects After Repeated-dose Oral Administration
}

Miguel Gama ( $\nabla$ fmgama@deb.uminho.pt)

University of Minho: Universidade do Minho https://orcid.org/0000-0002-5655-0015

Ana Cristina Rodrigues

University of Minho School of Engineering: Universidade do Minho Escola de Engenharia

Lígia Rodrigues

University of Minho School of Engineering: Universidade do Minho Escola de Engenharia

Sara Duarte-Silva

Universidade do Minho Escola de Medicina

Andreia Teixeira-Castro

Universidade do Minho Escola de Medicina

Nuno Lamas

Universidade do Minho Escola de Medicina

Fernando Dourado

Universidade do Minho Escola de Engenharia

\section{Research}

Keywords: Bacterial cellulose, repeated dose oral toxicity, cellulose intestinal absorption, cellulose toxicokinetics

Posted Date: December 3rd, 2021

DOI: https://doi.org/10.21203/rs.3.rs-1117993/v1

License: (c) (i) This work is licensed under a Creative Commons Attribution 4.0 International License. Read Full License 


\section{Abstract}

\section{Background}

Bacterial cellulose (BC) is a nanofibrillar polysaccharide produced by certain acetic acid bacteria. BC may be used in food, pharma and many other applications. However, detailed studies of the oral toxicology of $\mathrm{BC}$ are limited. Controversial data is published regarding this topic, specially when it comes to answering the question on whether cellulose is absorbed at the intestine.

\section{Methods}

Following the European Food Safety Authority guidelines (EFSA), this work presents the results of a 21-day repeated dose oral toxicity of BC in male and female Wistar Han rats (Wistar rats). In parallel, microcrystalline cellulose Avicel LM310 (commercially available as a food additive) was used.

Wistar rats were subjected to daily oral gavage of $0.75 \mathrm{~mL}$ of an aqueous suspension $1 \%(\mathrm{~m} / \mathrm{v})$ BC or of its counterpart of plant origin, Avicel LM310. Rats not submitted to gavage were included in the experiment as controls. Clinical observations, such as body weight measurements, food consumption and ophthalmologic evaluations were performed during the assay. After occision, serum chemistry, necropsic examination and histopathological analyses of the liver, kidneys, spleen and small and large intestines were performed. The presence of BC fibers along the gastrointestinal tract was assessed histologically using a Green Fluorescence Protein coupled to a Cellulose Carbohydrate Binding Module (GFP-CBM) from Clostridium cellulolyticum.

\section{Results}

No adverse clinical observations related to BC administration were noticed, nor appreciable differences in the toxicological endpoints evaluated were detected.

No evidence of BC persorption was found. Particularly, no BC was detected in the Peyer's patches or in the mesenteric lymphatic nodules. Moreover, the histopathological analyses revealed that the global architecture and morphology of the organs and tissues was preserved, among the different experimental groups, with no significant pathological changes among them. Regarding serum biochemistry, no significant differences were recorded, for both sexes.

\section{Conclusions}

These results demonstrate that BC nanofibers can be considered safe and, as the vegetal cellulose, can be used as a food additive.

\section{Background}

Colloidal plant cellulose and its derivatives have long been used as food ingredients (as dietary fibre) and additives (added functionality). The safety of these celluloses has been extensively reviewed by regulatory agencies, such as the US Food and Drug Administration (FDA), the European Food Safety Authority (EFSA), the Joint Committee of Experts from FAO/WHO on Food Additives (JEFCA) and the Selected Committee for Substances Generally Recognized as Safe (GRAS) (SCOGS) [1-7]. In early 80s, Turbak and co-workers [8-11] first proposed a list of applications for plant nanocellulose as a food additive. Given their dimensions, which may impart novel physical-chemical properties and behaviors and contribute to modulate digestion and absorption of nutrients, nanocelluloses have been increasingly investigated in the field. However, regulatory authorizations for their use in food are still missing, given that nano-scale materials often exhibit different properties and interactions with biological systems than those of their micro-sized counterparts [12-15]. Thus, investigating the toxicology of ingested nanomaterials, in particular nanocelluloses, is of great importance, when considering their market applications.

A few recent studies addressed already the safety aspects of nanocelluloses [16-21]. Mackie et al. [17] studied the gastrointestinal fate of cotton cellulose nano-crystals (CNCs) as Pickering stabilization agents in oil in water emulsions. The results showed that the CNCs were entrapped in the intestinal mucus layer and failed to reach the underlying epithelium. The study of DeLoid et al. [18] presents toxicological findings of NC (nanocellulose), from softwood bleached kraft fiber, in physiologically relevant in vitro and in vivo systems. In vitro studies employed a gastrointestinal tract simulator to digest two widelyused forms of NC, nanocellulose fibrils (NCF) and cellulose nanocrystals (CNC). A model of the small intestinal epithelium was used to assess their effects, over a period of $24 \mathrm{~h}$, on cell layer integrity, cytotoxicity and oxidative stress. Other than a 10\% increase over controls in reactive oxygen species (ROS) with $1.5 \% \mathrm{w} / \mathrm{w}$ CNC, no significant changes were observed. In vivo toxicity was evaluated in rats by "per os" administration, twice weekly, for five weeks, with $1 \%$ W/w suspensions of NCF. No significant differences in hematology, serum markers or histology were observed between controls and rats given CNF suspensions. These findings suggest that the ingested NC has little acute toxicity and is likely non-hazardous when ingested in small quantities. In another study, Andrade et al. [19] assessed the effects of NCF from the Heartof-peach palm in a male rat model (Rattus norvegicus albinus). The addition of NCF to the diet resulted in a trend for an increased weight of the animals. The blood sugar, triglycerides and cholesterol concentrations did not show any significant statistical difference over the whole duration of the experiment, indicating the maintenance of homeostasis of the animals. Moreover, Ong et al. [20] assessed the 90-day dietary toxicity, on Sprague Dawley rats, of fibrillated cellulose produced through mechanical homogenization of a wood pulp, as compared to Solka Floc, a conventional cellulose commonly used as a dietary fiber in animal feed and human dietary studies. Survival rate, clinical observations, body weight, food consumption, ophthalmologic evaluations, hematology, serum chemistry, urinalysis, post-mortem necroscopic evaluation with histopathology were performed. No adverse reactions were noted in relation to the administration of fibrillated cellulose. The no-observed-adverse-effect level (NOAEL) for fibrillated cellulose was $2194.2 \mathrm{mg} / \mathrm{kg} /$ day (males)and $2666.6 \mathrm{mg} / \mathrm{kg} /$ day (females), corresponding to the highest dose tested (4\%). These results demonstrate that the fibrillated cellulose behaves similarly to conventional cellulose and raises no safety concerns when used as a food ingredient at these concentrations. Another study by Chen et al., [21] aimed at determining the effects of subchronic oral NCF consumption on various health aspects of Western diets-fed mice. This work demonstrated that subchronic exposure to NCF at $30 \mathrm{mg} / \mathrm{kg}$ body weight produced some undesired health effects, including a decreased lean body mass and glucose homeostasis. This was assigned to the non-specific decrease intestinal absorption induced by NCF. 
Bacterial cellulose (BC) is an exopolysaccharide synthetized by Komagataebacter xylinus (formerly known as Gluconacetobacter xylinus), a strict aerobe Gram - negative bacteria. Although chemically identical to plant cellulose, BC presents some specific features. It can be obtained with higher purity, exhibiting higher degree of polymerization (800-11000) and crystallinity index. Under static culture, BC is a gelatinous film consisting of a 3D nanofibrillar network of pure cellulosic fibers. These randomly assembled ribbon-shaped fibrils are less than $100 \mathrm{~nm}$ wide, being composed of elementary nanofibrils aggregated in bundles with lateral sizes of 7-8 nm. These fibrils have several micrometers in length [22-29]. Due to its high aspect ratio and surface area, BC has superior water retention properties. While vegetable cellulose presents water retention values around $60 \%, \mathrm{BC}$ holds a water retention capacity up to $1000 \%$ of its dry mass. The unique structural, physicochemical, mechanical and biological features of BC have caught the attention as a versatile material for application in several areas, such as the biomedical, food, cosmetics, among others [30-34]. Particularly, it has been recognized as a gelling agent, thickener and suspension stabilizer with great potential for the food industry. Additionally, its potential role as an alternative source of dietary fiber has also been suggested [34].

Currently, BC is produced and marketed mainly in Asian countries under the trade name 'nata de coco' [35, 36]. The long history of BC consumption, without any reported cases of toxicity, suggests that $\mathrm{BC}$ is safe for consumption. Furthermore, some in vitro and in vivo studies assessing $\mathrm{BC}$ toxicity have demonstrated that it is not genotoxic, carcinogenic, pyrogenic or toxic for development or reproduction $[37,16,5]$. Moreover, BC does not induce acute or chronic oral toxicity or allergenicity $[37,16,5]$. In fact, the available data corroborate the general assumption that BC is non-toxic by ingestion or contact and does not trigger any inflammatory response or oxidative stress at the cellular level $[37,16,5]$. Some studies addressed the toxicology of BC. Hagiwara et al. [16] evaluated the effect of BC sub-acute administration to F344 rats for 28 days, at dietary levels of $0,1.25,2.5$, and $5.0 \%$. The treatment had no adverse effects on mortality, body weight, food and water consumption, urinalysis, ophthalmology, hematology, blood biochemistry and histopathology. At necropsy, slight increased absolute and relative cecum weights, evident in females ingesting $2.5 \%$ and $5.0 \%$ dietary levels, were considered to be a physiological adaptation to the poorly absorbed fermentation-derived cellulose. The non-observed-adverse-effect level (NOAEL) was 5,331 and 5,230 mg/kg body weights/day, for males and females, respectively. Schmitt et al. [5] tested the acute oral toxicity of a commercial product named Cellulon ${ }^{\circledR}$ fibre (dried BC:sucrose at a mass ratio of 1:1) in Sprague-Dawley rats. Rats were fed a single oral dosage of $2000 \mathrm{mg}$ Cellulon/Kg of body weight, via oral intubation, with no adverse treatment-related effects recorded. The sub-chronic toxicity of BC was investigated in Sprague-Dawley rats fed diets containing 0,5 , and $10 \%$ Cellulon ${ }^{\circledR}$ fibre or microcrystalline cellulose, for 13 weeks. There were no treatment-related deaths or clinical signs of toxicity. Body weight was unaffected. Food consumption was generally increased in treated animals (both Cellulon ${ }^{\circledR}$ fibre and microcrystalline cellulose), assigned to the relatively high-test article concentrations used. Statistically significant changes in haematology and clinical chemistry parameters were not considered significant. There were no notable gross pathologic or histopathological findings at necropsy and organ weights were unaffected. The outcomes were interpreted as proof that Cellulon ${ }^{\circledR}$ fibre and microcrystalline cellulose are toxicologically equivalent and that sub-chronic exposure to Cellulon ${ }^{\mathrm{TM}}$ fibre did not adversely affect these animals. The NOAEL was 7,000 and $8,500 \mathrm{mg} / \mathrm{kg} /$ day for male and female rats, respectively.

These studies did not assess whether celluloses are able to cross the intestinal epithelium. Several publications have reported the uptake of a variety of particles, including nano-sized ones, across the gastrointestinal tract in animal models [38-42]. With regards to plant cellulose (micro)particles, contradictory results on the hypothetical persorption phenomena have been reported over the last years $[1-3,43-45]$. Reports have shown that ${ }^{14} \mathrm{C}$ labelled plant cellulose [43] and BC [4] are slowly and limitedly degraded by the microflora of the large intestine of rats, resulting in metabolites that are partially absorbed by the colon. The absorbed cellulose degradation products were detected in the urine and on exhaled $\mathrm{CO}_{2} . \mathrm{ADME}$ (Absorption, Distribution, Metabolism, and Excretion) studies performed also with germ-free rats (without intestinal microflora) have shown total excretion of either plant or BC in the feces [4, 43], confirming that there is no absorption of non-degraded cellulose, but rather of its degradation products.

The present study was designed to assess the potential absorption and accumulation of BC at intestinal tract level, as well as any pathological changes in organs and tissues related with its potential toxicity, in male and female Wistar Han rats, following daily oral administration of BC for 21 days. The systemic toxicity of $\mathrm{BC}$ was also evaluated by measuring the serum standard parameters. After necropsy, to trace the fate of the administered BC, a CarbohydrateBinding Module (CBM3A) from Clostridium cellulolyticum coupled to a green fluorescent protein (GFP), was used for the histological visualization of cellulose, given its specificity and high affinity for cellulose, providing high detection sensitivity [6, 46-47].

\section{Results}

\section{Clinical observations and gross examination}

All rats survived until the end of the experimental period, showing no abnormal clinical signs nor gross pathological changes at necropsy that could be ascribed to $\mathrm{BC}$ or plant cellulose administration. The feces of all animals had normal consistency throughout the study. Compared to the control group (without gavage), at any given day, rats subjected to either BC or plant cellulose oral gavages showed no significant differences ( $p>0.05)$ in their measured body weight (Table 1). Also, when compared to the control, the daily oral gavages of both cellulose types had no effect $(p>0.05)$ on organ weight (expressed per animal bodyweight $x$ 100) for either sex. 
Table 1

Mean $( \pm S D$ ) bodyweight evolution and organ weight at necropsy of Wistar rats. The animals were subjected to daily gavage of bacterial or plant cellulose suspension $(0.75 \mathrm{~mL}, 1 \% \mathrm{~m} / \mathrm{v})$, during the 21 days of the experiment. The control

\begin{tabular}{|c|c|c|c|c|c|c|c|c|c|}
\hline & \multicolumn{6}{|c|}{ Animal Weight (g) } & \multicolumn{3}{|c|}{ Organ weight/animal bodyweight ( $\mathrm{gx} 100)$} \\
\hline & Group & Days: & 1 & 7 & 14 & 21 & Liver & Spleen & Kidney \\
\hline \multirow[t]{6}{*}{ Female } & Control & Mean & $170.8^{\mathrm{ab}}$ & 182.3 & $194.5^{\mathrm{a}}$ & $199.6^{\mathrm{b}}$ & 3.71 & 0.24 & 0.72 \\
\hline & & SD & 12.6 & 9.3 & 10.5 & 13.7 & 0.47 & 0.09 & 0.07 \\
\hline & BC & Mean & $180.7^{\mathrm{a}}$ & $185.25^{\mathrm{b}}$ & $196.3^{b}$ & $203.1^{\mathrm{a}}$ & 3.70 & 0.24 & 0.71 \\
\hline & & SD & 7.4 & 8.0 & 9.9 & 11.4 & 0.55 & 0.03 & 0.05 \\
\hline & PC & Mean & 186.3 & 193.0 & 200.5 & 203.0 & 3.48 & 0.20 & 0.74 \\
\hline & & SD & 33.6 & 26.8 & 22.1 & 21.4 & 0.19 & 0.01 & 0.03 \\
\hline \multirow[t]{6}{*}{ Male } & Control & Mean & 210.3 & 244.8 & 260.0 & 293.7 & 3.94 & 0.21 & 0.65 \\
\hline & & SD & 12.6 & 12.5 & 22.1 & 17.6 & 0.58 & 0.03 & 0.09 \\
\hline & $\mathrm{BC}$ & Mean & 223.5 & 250.5 & 279.8 & 293.0 & 3.81 & 0.20 & 0.66 \\
\hline & & SD & 11.6 & 12.8 & 16.0 & 20.8 & 0.55 & 0.03 & 0.05 \\
\hline & PC & Mean & $294.5^{a b c}$ & $308^{a}$ & $322.3^{b}$ & $332.8^{c}$ & 3.64 & 0.18 & 0.61 \\
\hline & & SD & 87.5 & 77.1 & 60.4 & 54.4 & 0.26 & 0.03 & 0.07 \\
\hline
\end{tabular}

\section{Assessment Of The Systemic Toxicity}

To assess the potential in vivo systemic toxicity of BC, blood and serum markers were analysed (Table 2). As compared to the control group, either plant or BC had no impact $(p>0.05)$ in blood glucose levels. The results of serum markers of liver function Total bilirubin (TBIL), alanine aminotransferase (ALT), aspartate transaminase (AST), and alkaline phosphatase (ALP) indicated no significant differences $(p>0.05)$ between bacterial and plant cellulose groups and the control group. The same was observed for the serum urea, creatine and electrolyte concentrations among all studied groups were noticed. The effect of BC on lipid metabolism was assessed by measuring the concentration of cholesterol ( $\mathrm{CHOL}$ ), with no significant differences ( $p>0.05)$ being observed among all experimental groups. 
Table 2

Mean $( \pm$ SD) serum biochemistry analysis of Wistar rats $(n=4)$. The animals were subjected to daily gavage of bacterial or plant cellulose suspensions $(0.75 \mathrm{r}$ shown. During the assay, food and water were provided to all animals ac

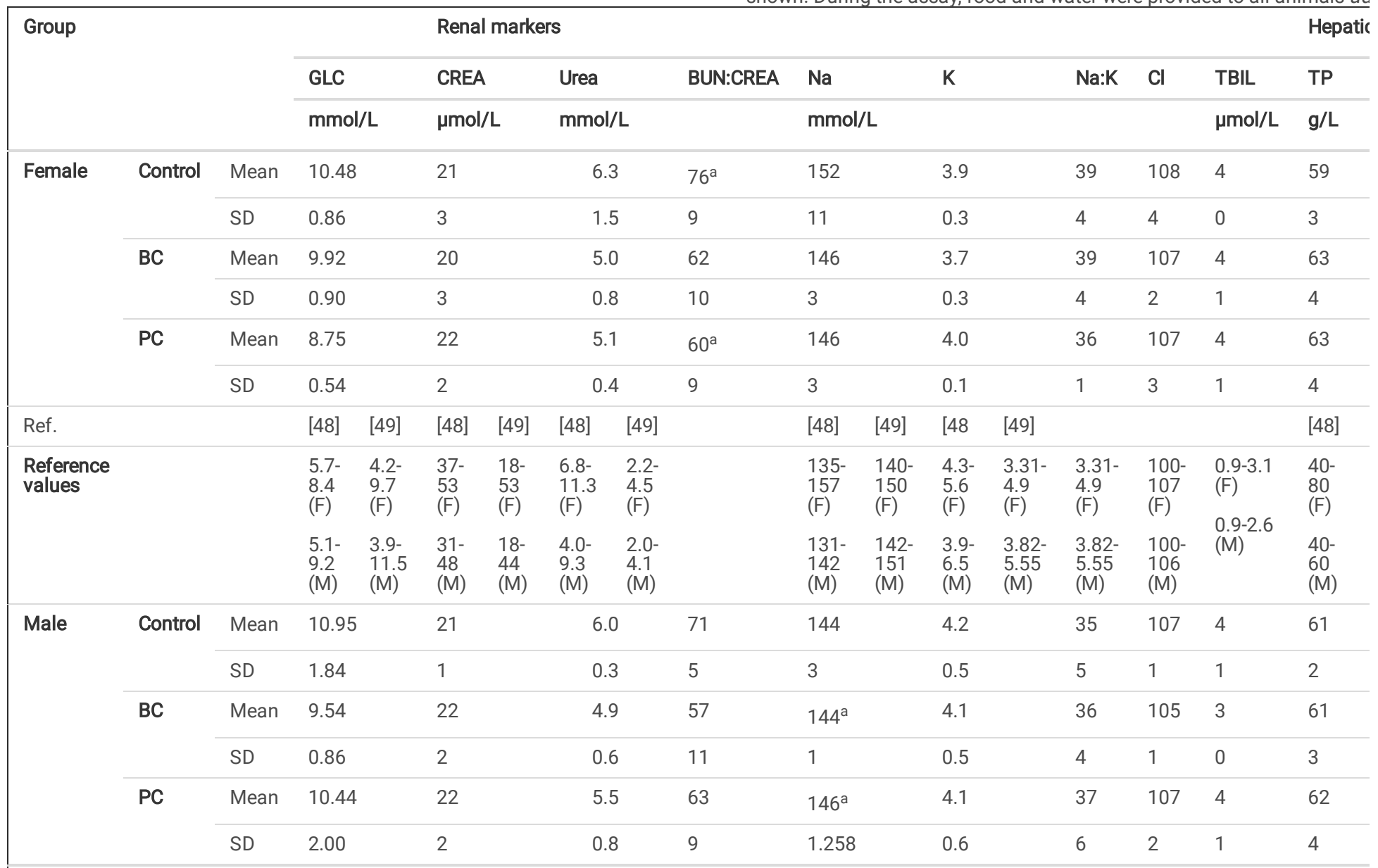

* For each column, superscripts with the same letter are statistically different $(p<0.05) \mathrm{F}$ - Female; M - Male

Alanine aminotransferase (ALT), albumin (ALB), alkaline phosphatase (ALP), aspartate aminotransferase (AST), total bilirubin (TBIL), blood urea nitrogen (BL $(\mathrm{GLU})$, osmolality, potassium (K), sodium (Na), total cholesterol (CHOL), total protein (TP)

Histopathological Analyses

Histological sections of the collected organs (liver, kidney and spleen) and the different regions of the intestinal tract of the animals were stained with H\&E and compared by an experienced pathologist, who was blind for the experimental groups. The comparative examination evidenced a global preservation of the organ architecture in all cases and the absence of significant pathological changes (Fig. 1). Regarding the intestinal sections, an apparent global maintenance of the organ normal architecture was observed with the absence of significant pathological changes, including inflammatory related alterations (Fig. 2). Also, no differences in histopathological analyses were detected between females and males from each experimental group.

\section{Cellulose Tracking}

Histological samples of the intestinal tract of all animals were stained with a CBM-GFP (green), to identify cellulose. Moreover, for actin-cytoskeleton and nucleus visualization the samples were also stained with Phalloidin-TRITC (red) and with DAPI (blue), respectively. In this experiment, two techniques were applied for the intestinal tissue preparation before freezing and cryosectioning: i) the swiss roll technique (SRT), which enables the observation of longitudinal sections of larger portions of the washed intestines, delivering more representative tissue sections for cellulose tracking as compared to the ii) gut bundling technique (GBT), which provides only transversal sections at specific regions of the rats' intestinal tract.

We first observed the distribution of cellulose in unwashed intestines using the GBT, also as a proof of concept of the cellulose staining method (representative images on Fig. 3). No cellulose was detected in intestinal associated lymphoid tissues - peyer patches and colonic patches (white arrows). Cellulose was observed only on the luminal space of intestinal sections (Fig. 3).

To better assess whether cellulose is able to cross the intestinal epithelium, the intestines were washed to remove, as much as possible, the cellulose present in the lumen and processed with GBT and SRT (representative images on Fig. 4 and 5, respectively). Negligible amounts of cellulose were detected on GBT washed intestinal samples; when detected cellulose was generally found in the luminal space or trapped between the villi or in Lieberkhun crypts. No cellulose was detected in intestinal associated lymphoid tissues - peyer patches and colonic patches (white arrows) (Fig. 4). Negligible amounts of cellulose were detected on SRT washed intestinal samples without any distinct pattern. No cellulose was detected in intestinal associated lymphoid tissues - Peyer patches (highlighted by white arrows) and colonic patches (Fig. 5). 
To demonstrate in more detail that cellulose did not penetrate the mucus layer of the intestinal tract, confocal microscopy images at higher magnification and at different z-planes (depth) were collected (Fig. 6). Cellulose fibers were detected on SRT washed ileum and colon sections trapped between the villi and in Lieberkhun crypts. No cellulose co-localizing with the intestinal absorptive epithelium or with lamina propria and submucosa was detected as can be observed in the different z-slices of the intestinal stained samples. In some cases, few discrete fragments of cellulose were found overlapping intestinal tissue.

Regarding the cellulose staining on serial histological samples of the mesenteric lymph nodes (MLN) from all animals, some discrete green dots were detected in all experimental groups (Fig. 7). This was observed with similar frequency in the positive and negative staining controls (omitting the incubation with CBMGFP conjugate), both showing a similar green diffusive signal.

\section{Discussion}

General clinical observations of the animals over the experiment, together with gross examination and further histopathological analysis of the collected organs (liver, kidneys, spleen and the different regions intestinal tract), demonstrate that cellulose did not exert any local pathological effect on the examined tissues, which have preserved their global structure and morphology without any detectable inflammatory infiltrates or other pathologically relevant changes (Table 1, Fig. 1 and 2).

The potential systemic toxicity of bacterial cellulose was assessed by serum markers analysis, taking plant cellulose and the control group (without gavage) as reference (Table 2). No significant differences $(p>0.05)$ were observed for either plant or BC. Total bilirubin, alanine aminotransferase, aspartate transaminase, and alkaline phosphatase are enzymes generally used to assess the liver function, with elevation in serum enzyme levels and TBIL being associated with liver damage [50]. Thus, apparently the different cellulose types have not compromised liver functions. The same conclusions stem from the analysis of the total protein (TP) values, which provide an estimation of the animals' nutritional status and assist on diagnosing several pathological conditions associated to liver and kidney disease [51]. A reduction in total protein (albumin and globulin), not observed in this work, is an indicator of an impaired hepatocellular function. The measurement of TBIL, serum urea and creatinine provides information on the likelihood of renal ailment or dysfunction [52]. As compared to the control groups no significant changes $(p>0.05)$ in urea and creatinine levels for both cellulose types, were detected, suggesting that $\mathrm{BC}$ did not impact the normal renal function. The effect of BC on lipid metabolism and predisposition to atherosclerosis was assessed by measuring the concentration of cholesterol ( $\mathrm{CHOL})$. None of the cellulose types had an impact $(p>0.05)$ in cholesterol levels [52].

It is important to note that the reference values established for biochemical tests, may not precisely represent those of a certain population or animal species and should, therefore, be carefully interpreted, once there is a wide range of physiological variation. These variations are influenced by environmental conditions, such as gender, age, origin, breeding system, feeding and lineage, which might have interfered with the obtained results [49,53]. According to the Scientific Committee for International Harmonization of Clinical Pathology Testing "the concurrent control data are more appropriate than historical reference ranges for comparison with test material groups" [49]. By analyzing the serum markers, we can note that depending on the reference source used, the obtained results may or not fall within the range of reference values. Nevertheless, no significant differences $(p>0.05)$ being noticed among groups, the results suggest that BC apparently does not exert any systemic toxicity, at least amenable to be detected by serum biochemistry analysis.

The cellulose tracking using Carbohydrate-Binding Module (CBM3A) from Clostridium cellulolyticum coupled to a green fluorescent protein (GFP) in different parts of the intestinal tract was performed. In unwashed intestines using the GBT (Fig. 3), the intestines of the control females exhibited higher cellulose content than the dosing groups, mainly in the jejunum, ileum and colon. For males, cellulose was detected in all experimental groups, mainly in the ileum and colon portions. The cellulose in the intestines of the control animals was ascribed to the type of diet supplied to the animals, which contains $4.1 \%$ crude fiber. In the males of the BC dose group, cellulose was detected in the lumen of the jejunum, ileum and in higher amounts in the colon, whereas for females, low amounts of cellulose were found. Since all animals grew normally and no relevant inter-group variations were found in serum biochemistry (section Assessment of the systemic toxicity), the observed differences in cellulose distribution may be due to inter-individual variability and to the (potentially variable) time gap between the last meal and euthanasia.

Unwashed intestinal histological sections processed by GBT have shown large amounts of cellulose, mainly in the ileum and colon regions. As expected, a significantly lower amount of cellulose was detected in the washed intestines, where cellulose was found only in a few cases, without any noticeable difference of frequency or location between the three experimental groups (Fig. 4 and 5). In such cases, it was located in the intestinal lumen or between the villi and in Lieberkuhn crypts (Fig. 6). Thus, intestine washing appears to have been incomplete, due to the intricate structure of the mucosa layer. From all of the collected images, no evidence of cellulose intestinal persorption (either bacterial or vegetal cellulose) was found in the lamina propria, the submucosa or in the gut associated lymphoid tissue. In particular, it never colocalized with the Peyer Patches, nor with colonic patches (Fig. 3-5; white arrows). The analysis of confocal microscopy images at higher magnification and at different z-planes (depths) (Fig. 6) showed that cellulose particles/fibres (either bacterial or plant) were confined to the Lieberkuhn cripts and between the intestinal villi. No internalized cellulose was detected in the intestinal absorptive epithelium nor in Peyer's patches or colonic patches (Fig. 6), confirming that neither bacterial or plant cellulose can penetrate the intestinal tract mucus layer and be absorbed.

Regarding the cellulose staining on serial histological samples of the mesenteric lymph nodes (MLN) from all animals, some discrete green dots were detected in all experimental groups (Fig. 7). This was observed with similar frequency in the positive and negative staining controls (omitting the incubation with CBMGFP conjugate), both showing a similar green diffusive signal. Therefore, this was assigned to autofluorescence of MLN tissues detected in green channel. Some chromophore molecules, such as proteins, nicotinic coenzymes, flavins and lipopigments, widely present in biological tissues, when excited by radiation of suitable wavelength, emit the so-called autofluorescence [54]. This has been previously observed in murine intestinal tissue sections [55], but to our knowledge there are no reports on autofluorescence of the rats' MLN. The rounded diffusive pattern of the observed autofluorescence signal is clearly distinguishable from the longer and sharper specific staining patterns observed with cellulose, which also exhibited a brighter staining. 
The intestinal absorption and translocation of microparticulate material, including cellulose, has been a longstanding study. Upon early publications in the 1960s on the persorption of ingested particulate cellulose and on the demonstration of its presence in the circulating blood stream, further research reported that microcrystalline particles ranging in size from 5-150 $\mu \mathrm{m}$ could be persorbed and detected in venous blood samples taken 1-2 hours after ingestion by rats, dogs, minipigs and in 1 human volunteer. Other studies suggested that, in single dose tests, persorbed particles are cleared from tissues within a few hours and they do not accumulate on repeated dosing even for several months [43].

Reaching the systemic dissemination would require a translocation route through the intestinal epithelium. Possible mechanisms of nanoparticle translocation are: (1) through the M-cells in the Peyer's patches; (2) through enterocytes by passive diffusion; (3) through enterocytes by transcytosis; (4) through the paracellular space [56]. As discussed by Koshani and Madadlou, 2018 [56] for cellulose nanocrystals, route 1 (through the M-cells in the Peyer's patches) is mostly employed for uncharged hydrophobic particles and includes an active transepithelial vesicular transport system from the lumen directly to lymphoid cells and tissues. The overall efficiency of this process is questionable since the population of $\mathrm{M}$ cell is $<1 \%$ of the intestinal epithelia. In this work, no cellulose particles were detected in the lymphoid tissues nor on the Peyer's patches. The passive transcellular transport mechanism (route 2 ) requires a high level of hydrophobicity as it involves partitioning into and diffusion across the cell plasma membrane. This path is normally restricted to small highly hydrophobic molecules, thus excluding cellulose. Endocytosis (route 3 ) is classified into phagocytosis and pinocytosis. The phagocytic cells, such as macrophages, typically internalize foreign materials with sizes larger than $0.5 \mu \mathrm{m}$, while pinocytosis occurs in all types of cells for a wide range of particle sizes from approximately $50 \mathrm{~nm}$ to $5 \mu \mathrm{m}$. Due to the low endocytic activity of enterocytes, active transcellular transport mechanism will play a minor role in the clearance of particles from the intestinal lumen. However, phagocytic activity similar to that demonstrated by enterocyte TLR4, promoting bacterial translocation across the intestinal barrier, may result in absorption. Route 4, the paracellular pathway, is also not likely to allow the pass-through of cellulose particles, because it is restricted to particles (molecules) measuring less than $5 \mathrm{~nm}$. BC presents randomly assembled ribbon-shaped fibrils with less than 100 $\mathrm{nm}$ in diameter. These are composed of elementary nanofibrils with lateral size of 7-8 nm, aggregated in bundles; these fibrils have several micrometres in length. Plant cellulose presents micrometer size [25-29]. Overall, the hypothetical absorption of cellulose particles fibers is thus confined to routes 1 and 3 and it may be expected to represent a very inefficient process. Indeed, the several studies addressing the fate of cellulose particles in the intestinal tract have found no evidences of translocation [1]. A recent study on the use cellulose nanocrystals as Pickering stabilizing agents in oil in water emulsions concluded that CNC were entrapped in the intestinal mucus layer and failed to reach the underlying epithelium. The authors concluded that CNC could be used safely as an effective emulsifier [57].

The intestinal absorption of the BC used in this work is particularly unlikely to occur, taking into account the size of the fiber's bundles obtained by disintegrating BC membranes produced by static culture. Most of the fully swollen fibers have at least few tenths of micrometers, spanning up to several hundred/thousands micrometers, as shown elsewhere [58]. Thus, while the persorption or translocation of microcrystalline cellulose is still a matter of debate, the occurrence of this process for such large BC fiber bundles is rather doubtful. On the other hand, it must be considered that cellulose is partially hydrolyzed by the flora in the gut, although this is a process that occurs to larger extent in the rat than in humans [59]. In this case, a size reduction may occur along with degradation. Consequently, it may be speculated that smaller particles may be generated and translocated more efficiently. In that case, however, it may be questioned whether BC, microcrystalline cellulose and the cellulose naturally present in food will, once partially degraded, be much different from each other. The cellulose naturally occurring in food, and specially the pure cellulose already used as food additive, through degradation, must undergo a comminution process and give rise to cellulose particles with a widespread range of sizes and shapes (for example in colloidal celluloses, from FMC Biopolymers, commercially used as food additives, $99 \%$ of the particles are $<1 \mu \mathrm{m}[60,61])$. While we did not find evidence of cellulose translocation or accumulation in the gut lymphoid tissue, we speculate that the low efficiency translocation process - if it occurs to some extent - would occur as well with any other cellulose source present in food, as it undergoes degradation, in case this occurs to significant extent.

\section{Methods}

\section{Production of bacterial cellulose}

BC was obtained by static culture fermentation with Komagataeibacter sucrofermentans ATCC 700178 (from the American Type Culture Collection) as described in Rodrigues et al. [62]. Briefly, the culture medium was composed by $40 \mathrm{~g} / \mathrm{L}$ molasses, $7 \mathrm{~g} / \mathrm{L}$ Corn Steep Liquor, $0.5 \%$ ( $\mathrm{m} / \mathrm{v}$ ) ammonium sulphate, $2.7 \mathrm{~g} / \mathrm{L}$ disodium hydrogen phosphate, $1.15 \mathrm{~g} / \mathrm{L}$ citric acid and $1.5 \%(\mathrm{v} / \mathrm{v})$ of ethanol. Culture medium pH was adjusted to 5.5 . The growth culture (inoculum) was incubated for $2 \mathrm{~d}$ at $30{ }^{\circ} \mathrm{C}$ under static conditions. Afterwards, it was transferred to sterile containers with fresh culture medium, making up $10 \%$ of their final volume. The containers were incubated in static conditions for 10 days at $30^{\circ} \mathrm{C}$. After cultivation, the obtained $\mathrm{BC}$ was purified using $0.1 \mathrm{M}$ sodium hydroxide, $1 \%(\mathrm{v} / \mathrm{v})$ Divosan Hypochlorite and water, each step lasting 24h, at room temperature. Then, BC was ground into a pulp using a blender and concentrated through centrifugation at $3667 \mathrm{xg}$ for 30 minutes, to $5 \%(\mathrm{~m} / \mathrm{v})$ final solids and stored at $4{ }^{\circ} \mathrm{C}$, until use.

\section{Compliance with ethical and procedural standards for animal experimentation}

The study was performed at Instituto de Investigação e Inovação em Saúde (I3S), Universidade do Porto (Porto, Portugal). The experimental procedures followed the EU Directive 2010/63/EU and National Decreto-Lei 113/2013 legislation for animal experimentation and welfare. The rats' housing, handling and experimentation were accredited by the Portuguese National Authority for Animal Health, Direção-Geral de Alimentação e Veterinária (DGAV) (approval ${ }^{\circ}$ 012910/2020-08-[7]. Although, the experimental design deviated in some points from the OECD Guideline 408, such as the duration, amount of test substance, timeframe and bloodwork analyses, its execution fully complied with the quality standards.

\section{Animals, housing and feeding conditions}


Eight weeks old Wistar Han IGS Rats [Crl:WI(Han)] (Wistar Rats), twelve males and twelve females, were used in this study. The rats were obtained from Charles River (Barcelona, Spain) and bread at I3S. On arrival the animals were examined for signs of health status, followed by a one-week adaptation period. They were kept under controlled environmental conditions for one week before starting the experiment. Afterwards, each male and female rat was randomly allocated in groups of 2 to compose the different experimental groups. After allocation, each rat was uniquely numbered with a color marker in their tail and placed in polycarbonate type III H cages with a stainless-steel wire lid and a polysulphone filtertopcage (Tecniplast), with corncob and carboard tubes as bedding materials. An artificial light/dark cycle with a sequence of $12 \mathrm{~h}$ was applied. The room was ventilated with about $15-20$ air changes per hour. A temperature of $22 \pm 2{ }^{\circ} \mathrm{C}$ and a relative humidity of $55 \pm 15 \%$ was maintained.

Food and water were provided ad libitum to all animals during the experiment. They received a commercial 2014S Teklad rodent diet (Teklad diets, ENVIGO) based on $14.3 \%$ crude protein, $4 \%$ fat, $48 \%$ carbohydrates and $4.1 \%$ crude fiber in addition to vitamins and fatty acids.

\section{Test substance and dosing concentration}

Given the high viscosity of $\mathrm{BC}$, the aqueous concentration for daily oral gavage was adjusted to $1 \%(\mathrm{~m} / \mathrm{v})$ - as measured by gravimetry after drying overnight at $105^{\circ} \mathrm{C}$. The ground $\mathrm{BC}$ suspension was then sterilized by autoclaving for 20 minutes, at $120^{\circ} \mathrm{C}$ and $1 \mathrm{bar}$.

In parallel, $1 \%(\mathrm{~m} / \mathrm{v})$ aqueous suspension of commercial plant cellulose (PC), Avicel LM310 (kindly provided by FMC Biopolymers) was also prepared and sterilized.

All the cellulose suspensions were prepared beforehand and stored at $4{ }^{\circ} \mathrm{C}$. Before oral administration, the suspensions were warmed to room temperature and homogenized by vigorously vortex agitation.

\section{1-day repeated-dose toxicity study}

The study comprised two dose groups and one control group, each having four rats/sex (Table 3). Each rat from the dosing groups received a single dose of $0.75 \mathrm{~mL}$ of $1 \%$ cellulose suspension (plant or bacterial), daily, for a period of 21 days. The administration was performed in the morning at a fixed time by oral gavage, using a polypropylene gavage needle of $1.3 \times 1.3 \mathrm{~mm}$ without ball tip. The control group did not receive oral gavage.

Table 3 Test groups and substances administered.

\begin{tabular}{|lll|}
\hline Group & Test substance (21 days) & Nr. animals \\
\hline Control & No gavage & 4 male +4 female \\
BC & $1 \%$ Bacterial cellulose; $0.75 \mathrm{~mL} /$ day & 4 male +4 female \\
\hline PC & $1 \%$ Plant Cellulose - (Avicel LM 310$) ; 0.75 \mathrm{~mL} /$ day & 4 male +4 female \\
\hline
\end{tabular}

The animals' well-being was observed daily, and all the cages were inspected for possible deaths or moribund animals, twice daily. Detailed clinical observations were conducted prior to the first exposure to the compounds (pre-dose) and once a week thereafter. All animals were assessed for the following clinical signs: changes in the eyes, skin, fur, mucous membranes, secretions and excretions, autonomic activity and changes in their gait. Their individual body weight was recorded at start of the treatment (day 0), in weekly intervals thereafter, and before the euthanasia. By the end of experiment, the animals were deeply anesthetized with gaseous isoflurane in 0.2-0.3 L/min $\mathrm{O}_{2}$, and terminal blood was collected by intracardiac puncture into CAT serum Sep Clot Activator tubes $\left(\right.$ VACUETTE $\left.^{\circledR}\right)$ for analyses of the biochemical parameters. The blood samples collected for biochemistry were allowed to clot and the serum was obtained by centrifugation at $5000 \mathrm{xg}$ for 10 minutes. All animals were subjected to gross necropsy.

\section{Clinical biochemistry tests}

Serum markers analysis was performed at the Instituto de Ciências Biomédicas Abel Salazar (ICBAS) da Universidade do Porto (Porto, Portugal), using the IDEXX equipment, Catalyst One Chemistry Analyzer. The assessed parameters comprised alanine albumin (ALB), aminotransferase (ALT), alkaline phosphatase (ALP), aspartate aminotransferase (AST), total bilirubin (TBIL), blood urea nitrogen (BUN), chloride (Cl'), creatinine (CREAT), globulin (GLOB), glucose $(\mathrm{GLU})$, osmolality, potassium $\left(\mathrm{K}^{+}\right)$, sodium $\left(\mathrm{Na}^{+}\right)$, total cholesterol $(\mathrm{CHOL})$, total protein $(\mathrm{TP})$ and urea.

\section{Gross examination and histopathology}

Following euthanasia, the kidneys, liver and spleen from all animals were collected, trimmed off of any adherent tissue for necropsy examination and weighted. The animals' intestinal tract was also collected and processed by two different procedures [63] (Table 4), described in section Cellulose trackingintestinal-tract histological processing.

The collected organs were fixed with 4\% paraformaldehyde (PFA) and processed for paraffin-embedding in an automated tissue processor (Leica EG140C Pathology Embedding Center Paraffin Dispenser). Paraffin embedded specimens were cut in $5 \mu$ m-thick sections (MICROM HM325) and stained with hematoxylin \& eosin (H\&E), using an automated stainer (LEICA ST5010 Autostainer XL). Also, histological samples of the different regions of the intestinal tract from the different experimental groups were subjected to H\&E staining. All H\&E-stained samples were visualized using an optical microscope (BX61 
Olympus) coupled to a DP74 digital camera (Olympus) and using bright field. Images were acquired using CellSens Imaging Software Version 1.16 (Olympus). The histopathological analysis was blindly conducted by an independent experienced pathologist.

\section{Cellulose tracking -intestinal-tract histological processing}

\section{Gut bundling technique (GBT)}

The GBT was adopted for half of the females and males from each experimental group (Table 4). The small and large intestines were exposed and collected. Afterwards, they were cut into three equal segments to obtain the duodenum, jejunum, and ileum regions. The distal colon and the mesenteric lymph nodes (MLN) from each animal were also collected. For one female and male of each experimental group, the lumen of each small and large intestinal segment was washed with phosphate buffer saline (PBS), while for the other female and male, the collected intestines were not washed. All the collected intestinal segments and the mesenteric lymph nodes were embedded in OCT (Optimal Cutting Temperature) compound (Tissue-Tek ${ }^{\circledR}$, SakuraTM, The Netherlands), frozen using isopentane in liquid nitrogen and cryo-sectioned in $20 \mu \mathrm{m}$-thick sections using a cryostat (LEICA CM 1900).

\section{Swiss roll technique (SRT)}

For the remaining animals of each group, two males and two females (Table 4), the small intestines were collected and cut into three equal segments to obtain the duodenum, jejunum, and ileum regions (as with the GBT). Similarly, the distal colon was also collected, as well as the mesenteric lymph nodes from each animal. The lumen of each small and large intestine-portion was washed with PBS, cut longitudinally, opened so that the lumen is facing upward and then rolled. All these tissues were embedded in OCT compound (Tissue-Tek®, SakuraTM, The Netherlands), frozen using isopentane in liquid nitrogen and cryo-sectioned in $20 \mu$ m-thick sections using a cryostat (LEICA CM 1900).

Table 4 Histological processing methods for observation of the rats' intestinal tract.

\begin{tabular}{|c|c|c|c|c|c|}
\hline \multirow{2}{*}{$\begin{array}{l}\text { Group } \\
\# \mathbf{~}\end{array}$} & \multicolumn{2}{|c|}{$\mathrm{N}^{\circ}$ animals } & \multicolumn{3}{|l|}{ Technique } \\
\hline & $4 M+4 F$ & $2 M+2 F$ & Gut bundling & $1 \mathrm{M}+1 \mathrm{~F}$ & Washed \\
\hline & & & & $1 M+1 F$ & Unwashed \\
\hline & & $2 \mathrm{M}+2 \mathrm{~F}$ & Swiss roll & $2 \mathrm{M}+2 \mathrm{~F}$ & \\
\hline
\end{tabular}

i -test group (Control, BC and PC); M - male; F - female;

\section{Staining and microscopic observations}

To assess the intestinal fate of cellulose, all collected samples from the distinct regions of the intestinal tract and the MLNs were subjected to cellulose staining using a Carbohydrate-binding module (CBM3A from Clostridium cellulolyticum) coupled to a green fluorescent protein (CBM-GFP) (NZYTech Ltd), as previously described with some modifications [64]. Briefly, the samples were fixed with a 4 \% PFA solution for 30 min, followed by a washing step with PBS buffer. Permeabilization was carried out with $0.5 \%$ Triton X-100 in PBS at room temperature, in a humid chamber; then the blocking step was performed with $10 \%(\mathrm{v} / \mathrm{v}$ ) fetal calf serum (FCS) in PBS for 1h. For actin-cytoskeleton staining, the slides were incubated with Phalloidin-TRITC (1/500 dilution from a stock solution $0.5 \mathrm{mg} / \mathrm{mL}$ ) (Sigma- Aldrich) for $1 \mathrm{~h}$ in a humid chamber. Then, the samples were washed with PBS (three times, 3 min each) and incubated with CBM-GFP (1/20 dilution from a stock solution $1 \mathrm{mg} / \mathrm{mL})$ for $2 \mathrm{~h}$ at room temperature in a humid chamber. Sections were washed again with PBS and incubated with DAPI (1/1000 dilution from a stock solution $1 \mathrm{mg} / \mathrm{mL}$ ) (Thermo Scientific) for $10 \mathrm{~min}$, for nucleus visualization. Finally, the slides were washed with PBS (three times; 5 min each) and mounted with permafluor mounting media (Thermo Scientific). Fluorescent staining was visualized using a fluorescence microscope (BX61, Olympus) coupled to a DP74 digital camera (Olympus), using a set of three filters (DAPI 360-370/420nm Olympus U-MNU2; GFP 425-440/505-540nm, Chroma 31019; TRITC 530-550/575nm, Olympus MWIG3). Images were acquired using Cel/Sens Imaging Software Version 1.16 (Olympus). Confocal images of fluorescent stained histological samples were also acquired using a Laser Scanning Confocal Microscope (FluoView 1000 , Olympus) with a $60 x$ oil, 1.35 NA objective lens. Z-stack images were acquired using the following laser lines: $405 \mathrm{~nm}$ laser (for DAPI), $488 \mathrm{~nm}$ laser (for GFP fluorophore) and $559 \mathrm{~nm}$ (for TRITC). Confocal pinhole was adjusted to 1.0 Airy unit of optical slice, and z-stacks were acquired every $0.5 \mu \mathrm{m}$ between each zsection. Images were acquired with the software FV10-Ver4.1.1.5 (Olympus).

\section{Statistical analysis}

The obtained raw data were statistically analyzed using Graph Pad Prism software (version 5.01, windows). The values were expressed as the mean \pm standard deviation of the mean (SD). Differences in data of body weight and body weight gain, organ weight, hematological and clinical biochemistry were statistically analyzed using Two-way repeated measure ANOVA, with Geisser-Greenhouse correction and Tukey post hoc test. All analyses and correlations were assessed at $95 \%$ level of confidence $(p<0.05)$.

\section{Conclusions}

The herein presented results demonstrate that bacterial cellulose does not undergo intestinal absorption nor does it exert any local adverse effects on the gastrointestinal tract. The systemic assessment of toxicity, conducted by measuring several serum markers, revealed that the consumptions of $\mathrm{BC}$ had no 
impact $(p>0.05)$ in blood biochemistry of Wistar rats. Observation of histological samples of the different regions of the rats' intestinal tract and mesenteric lymph nodes did not show any local effects from the consumption of BC. Also, BC was not detected in the Peyer's and colonica patches and the mesenteric lymphatic nodules. Data gathered in this study strongly indicates that the intestinal absorption of bacterial cellulose is particularly very improbable to occur and thus, its consumption is implausible to cause any toxicological effects.

\section{Abbreviations}

ADME: Absorption, Distribution, Metabolism, and Excretion

AF: autofluorescence

ALB: albumin

ALP: alkaline phosphatase

ALT: alanine aminotransferase

AST: aspartate transaminase

BC: Bacterial cellulose

BUN: blood urea nitrogen

CBM: Carbohydrate Binding Module

CHOL: total cholesterol

Cl: chloride

CNC: cellulose nanocrystals

CNCs: cotton cellulose nano-crystals

CREAT: creatinine

DAPI: 2-(4-Amidinophenyl)-6-indolecarbamidine dihydrochloride

DGAV: Direção-Geral de Alimentação e Veterinária

EFSA: European Food Safety Authority guidelines

FDA: US Food and Drug Administration

GBT: gut bundling technique

GFP: green fluorescent protein

GGT: -glutamyl transferase

GLOB: globulin

GLU: glucose

H\&E: Hematoxylin and Eosin

JEFCA: Joint Committee of Experts from FAO/WHO on Food Additives

K: potassium

Na: sodium

NC: nanocellulose

NCF: nanocellulose fibrils

NOAEL: no-observed-adverse-effect level

OCT: Optimal Cutting Temperature

Phalloidin- TRITC: Phalloidin-Tetramethylrhodamine B isothiocyanate 
PC: plant cellulose

PFA: paraformaldehyde PFA

ROS: reactive oxygen species

SCOGS: Selected Committee for Substances Generally Recognized as Safe (GRAS)

SD: standard deviation of the mean

SRT: swiss roll technique

TBIL: Total bilirubin

TP: total protein

\section{Declarations}

\section{Ethics approval and consent to participate}

All experimental procedures followed the handling guidelines established the EU Directive 2010/63/EU and National Decreto-Lei $113 / 2013$ legislation for animal experimentation and welfare and permits from the Portuguese National Authority for Animal Health, Direção-Geral de Alimentação e Veterinária (DGAV) (approval nº 012910/2020-08-07). Prior to the study, the experimental protocols were approved by the local Animal Ethics Council.

\section{Consent for publication}

Not applicable.

\section{Availability of data and materials}

The representative summary data of the analyzed datasets within this study were provided in the manuscript. However, all of the collected results can be made available by the corresponding author, upon reasonable request.

\section{Competing interests}

The authors declare that they have no competing interests

\section{Funding}

This study was supported by the Portuguese Foundation for Science and Technology (FCT) under the scope of the strategic funding of UIDB/04469/2020 unit.

\section{Authors" contributions}

ACR and LR these authors contributed equally to this work, contributed to experimental design, performed experiments, data analysis and co-wrote manuscript.

SD-S and AT-S these authors provided the cryostat to cryo-sectioned of intestine-portion using a and provide the equipment and conditions to microscopic observations.

NL experienced pathologist performed the histopathological analyses of the animals' collected organs (liver, kidney and spleen) and the different regions of the intestinal tract.

FD and MG these authors contributed equally to this work, contributed to experimental design, data analysis and prepared the first version of manuscript with input from all authors.

All authors contributed to the writing of the manuscript. All authors read and approved the final manuscript.

\section{Acknowledgements}

The authors thank the institutions and workers who participated in the study. 


\section{Authors' information}

\section{Affiliations}

CEB - Centre of Biological Engineering, University of Minho, Campus Gualtar, 4710-057 Braga, Portugal

Ana Cristina Rodrigues, Lígia Rodrigues, Fernando Dourado and Miguel Gama

Life and Health Sciences Research Institute (ICVS), School of Medicine, University of Minho, Campus Gualtar, 4710-057, Braga, Portugal

Sara Duarte-Silva \& Andreia Teixeira-Castro and Nuno Lamas

ICVS/3B's, PT Government Associate Laboratory, Braga/Guimarães, Portugal.

Sara Duarte-Silva \& Andreia Teixeira-Castro and Nuno Lamas

Anatomic Pathology Service, Pathology Department, Centro Hospitalar e Universitário do Porto, Largo Professor Abel Salazar, 4099-001, Porto, Portugal.

Nuno Lamas

\section{Corresponding authors}

Correspondence to Miguel Gama,

CEB- Centre of Biological Engineering, University of Minho, Campus de Gualtar 4710-057 Braga, Portugal, E-mail: fmgama@deb.uminho.pt, Telf.: + 351 253 604 400; fax: +351253678986

\section{References}

1. Kotkoskie LA, Butt MT, Freeman C, Weiner ML. Qualitative investigation of uptake of fine particle size microcrystalline cellulose following oral administration in rats. J Anat. 1996; 189(Pt 3): 531-535.

2. Nagele W, Muller N, Brugger-Picher E. Nagele, J. Persorption Of Plant Microparticles After Oral Plant Food Intake. The Internet Journal of Herbal and Plant Medicine. 2013; 2(1): 1-8.

3. Kelleher J, Walters M P, Srinivasan T R, Hart G, Losowsky M S. Degradation of cellulose within the gastrointestinal tract in man. Gut. $1984 ; 25(8)$ : 811.

4. Juhr N C and J Franke. A method for estimating the available energy of incompletely digested carbohydrates in rats. J Nutr, 1992; 122(7): 1425-1433.

5. Schmitt DF, Frankos VH, Westland J, Zoetis T. Toxicologic evaluation of Cellulon (TM) fiber: Genotoxicity, pyrogenicity, acute and subchronic toxicity. J Am Coll Toxicol, 1991; 10(5): 541-554.

6. Knudsen KB, Kofoed C, Espersen, R, Højgaard C, Jakob RW, Willemoës M, et al., Visualization of Nanofibrillar Cellulose in Biological Tissues Using a

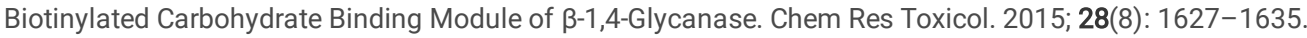

7. OECD, Test No. 408: Repeated Dose 90-Day Oral Toxicity Study in Rodents. 2018.

8. Turbak, AF, et al., US 4.341.807. 781 Washington, DC: US: Patent and Trademark Office, 1982.

9. Turbak AF, Snyder FW, Sandberg KR. Microfibrillated cellulose, a new cellulose product: Properties, uses, and commercial potential. J Appl Polym Sci: Appl Polym Symp. 1983a; 37: 815-827.

10. Turbak AF, et al. US 4.378.381. Washingtn DC: US: Patent and Trademark Office. 1983b.

11. Turbak AF, et al. US 4.487.634. 788 Washington, DC: US: Patent and Trademark Office. 1984.

12. Gómez CH, Serpa A, Velásquez-Cock J, Gañán P, Castro C, Vélez L, Zuluaga Z. Vegetable nanocellulose in food science: A review. Food Hydrocoll. 2016; 57: $178-186$.

13. Endes C, Camarero-Espinosa S, Mueller S, Foster EJ, Petri-Fink A, Rothen-Rutishauser B, et al. A critical review of the current knowledge regarding the biological impact of nanocellulose. J Nanobiotechnol. 2016. 14(78):1-14.

14. Ma T, Hu X, Lu S, Liao X, Song Y, Hu X, Nanocellulose: a promising green treasure from food wastes to available food materials. Crit Rev Food Sci Nutr. 2020; 1-14.

15. Shatkin JA, Kim B. Cellulose nanomaterials: life cycle risk assessment, and environmental health and safety roadmap. Environ Sci Nano. 2015; 2: 477499.

16. Hagiwara A, Imai N, Sano M, Kawabe M, Tamano S, Kitamura S, et al. A 28-day oral toxicity study of fermentation-derived cellulose, produced by Acetobacter aceti subspecies xylinum, in F344 rats. J Toxicol Sci. 2010; 35(3): 317-325.

17. Mackie A, Gourcy S, Rigby N, Moffat J, Capron I, Bajka B. The fate of cellulose nanocrystal stabilised emulsions after simulated gastrointestinal digestion and exposure to intestinal mucosa, Nanoscale. 2019;11:2991-2998.

18. DeLoid G, Cao X, Molina RM, Silva DI, Bhattacharya K, Ng KW et al., Toxicological effects of ingested nanocellulose in in vitro intestinal epithelium and in vivo rat models. Environ Sci Nano. 2019; 6: 2105-2115. 
19. Andrade DRM, Mendonça MH, Helm CV, Magalhães WLE, de Muniz GIB, Kestur SG. Assessment of Nano Cellulose from Peach Palm Residue as Potential Food Additive: Part II: Preliminary Studies. J Food Sci Technol. 2014; 52(9): 5641-5650.

20. Ong KJ, Ede JD, Pomeroy-Carter C A, Sayes CM, Mulenos MR, Shatkin JA. A 90-day dietary study with fibrillated cellulose in Sprague-Dawley rats. Toxicol Rep. 2020; 7: 174-182.

21. Chen Y, Lin YJ, Nagy T, Kong F, Guo TL. Subchronic exposure to cellulose nanofibrils induces nutritional risk by non-specifically reducing the intestinal absorption. Carbohydr Polym. 2020; 229(9):115536.

22. Masaoka M, Ohe T, Sakota N. Production of Cellulose from Glucose by Acetobacter xylinum. J Ferment Bioeng. 1993; 75(1): 18-22.

23. Shoda M, Sugano Y. Recent advances in bacterial cellulose production. Biotechnol Bioprocess Eng. 2005; 10: 1-8.

24. Jonas R, Farah LF. Production and application of microbial cellulose. Polym Degrad Stab 1998; 59: 101-106.

25. Brown Jr RM, Willison JH, Richardson CL. Cellulose biosynthesis in Acetobacter xylinum: visualization of the site of synthesis and direct measurement of the in vivo process, Proced Natl Acad Sci U.S.A. 1976; 73(12): 4565-4569.

26. Wanichapichart P, Kaewnopparat S, Buaking K, Puthai W. Characterization of cellulose membranes produced by Acetobacter xyllinum, Songklanakarin. J Sci Technol. 2002; 855-862.

27. Lin K, Lin H. Quality characteristics of Chinese-style meatball containing bacterial cellulose (nata). J Food Sci. 2004; 69(3): SNQ107-SNQ111.

28. Oliveira RL, Barud H da S, de Assunção RMN, Meireles C da S, Carvalho G O, Filho GR, et al. Synthesis and characterization of microcrystalline cellulose produced from bacterial cellulose. J Therm Anal Calorim. 2011; 106 (3):703-709.

29. Iguchi M, Yamanaka S, Budhiono A, Bacterial cellulose-a masterpiece of nature's arts, J. Mater. Sci. 2000; <background-color:\#FFCC66;bverticalalign:super;>35</background-color:\#FFCC66;bvertical-align:super;>(2): 261-270.

30. Jonas R, Farah F. Production and application of microbial cellulose. Polym. Degrad. Stab. 1998; 101-106.

31. Klemm D, Schumann D, Udhardt U, Marsch S. Bacterial synthesized cellulose e artificial blood vessels for microsurgery. Prog Polym Sci. 2001; 15611603.

32. Klemm D, Heublein B, Habil H-P F, Bohn A. Cellulose: fascinating biopolymer and sustainable raw material. Angew Chem Int Ed England. 2005; 33583393.

33. Ullah H, Santos, HA, khan T. Applications of bacterial cellulose in food, cosmetics and drug delivery. Cellulose. 2016 ; 23 (4): $2291-2314$.

34. Dourado F, Leal M, Martins D, Fontão A, Rodrigues AC, Gama M, Chapter 7- Cellulose as Food Ingredients/Additives: Is there a Room for BNC?, In Bacterial Nanocellulose: from biotechnology to bio-economy. Gama M, Bielecky S, Dourado F, editors. Elsevier. Amsterdam: Netherlands; 2016. p.123-133. ISBN: 978-0-444-63458-0.

35. Piadozo Ma ES, Chapter 13- Nata de Coco Industry in the Philippines, In Bacterial Nanocellulose: from biotechnology to bio-economy. Gama M, Bielecky S, Dourado F, editors. Elsevier. Amsterdam: Netherlands; 2016. p. 123-133. ISBN: 978-0-444-63458-0.

36. Phisalaphong M, Tran T-K, Taokaew S, Budiraharjo R, Febriana GG, Nguyen D-N et al., Chapter 14- Nata de coco Industry in Vietnam, Thailand, and Indonesia, In Bacterial Nanocellulose: from biotechnology to bio-economy. Gama M, Bielecky S, Dourado F, editors. Elsevier. Amsterdam: Netherlands; 2016. p. 231-236. ISBN: 978-0-444-63458-0.

37. Dourado F, Gama M, Rodrigues AC. A review on the toxicology and dietetic role of bacterial cellulose. Toxicol Rep. 2017 ; 4: 543-553.

38. O'Hagan DT. The intestinal uptake of particles and the implications for drug and antigen delivery. J Anat. 1996; 189(Pt 3): 477-482.

39. Hillyer JF, Albrecht RM. Gastrointestinal persorption and tissue distribution of differently sized colloidal gold nanoparticles. J Pharm Sci. 2001; 90(12): 1927-36.

40. Florence AT. The Oral Absorption of Micro- and Nanoparticulates: Neither Exceptional Nor Unusual. Pharm Res. 1997; 14(3): $259-266$.

41. Schleh C, Semmler-Behnke M, Lipka J, Wenk A, Hirn S, Schäffler M, et al. Size and surface charge of gold nanoparticles determine absorption across intestinal barriers and accumulation in secondary target organs after oral administration. Nanotoxicology. 2012; 6(1): 36-46.

42. Kim KS, Suzuki K, Cho H, Youn Y S, Bae YH. Oral Nanoparticles Exhibit Specific High-Efficiency Intestinal Uptake and Lymphatic Transport. ACS Nano. 2018; 12(9): 8893-8900.

43. JEFCA. Re-evaluation of celluloses E 460(i), E 460(ii), E 461, E 462, E 463, E 464, E 465, E 466, E 468 and E 469 as food additives. EFSA Journal; 2018: 1104.

44. JEFCA. Safety evaluation of certain food additives and contaminants. Microcrystalline cellulose (WHO Food Additives Series 40). The forty-ninth meeting of the Joint FAO/WHO Expert Commitee on Food Additives, in World Health Organization Technical Report Series. 1998; 1-22.

45. Joint FAO/WHO Expert Committee on food Additives. Evaluation of certain food additives and contaminants: forty-ninth report of the Joint FAO/WHO Expert Committee on Food Additives. World Health Organization: Geneva. 1999.

46. Ong E, Gilkes NR, Miller Jr RC, Warren RA, Kilburn DG. The cellulose binding domanin (CBD (Cex)) of an exoglucanase from Cellulomonas fimi: production Escherichia coli and

47. characterization of the polypeptide. Biotechnol Bioeng. 1993; 42: 401-409. https://.Accessed 1 October 2021.

48. Mythilypriya R, Shanthi P, Sachdanandam P. Oral Acute and Subacute Toxicity Studies with Kalpaamruthaa, a Modified Indigenous Preparation, on Rats. J Health Sci. 2007; 53(4): 351-358.

49. Ozer J, Ratner m, Shaw M, Bailey W, Schomaker S. The current state of serum biomarkers of hepatotoxicity. Toxicol. 2008; 245(3): 194-205.

50. Sunmonu TO, Afolayan AJ. Protective effect of Artemisia afra Jacq. on isoproterenol induced myocardial injury in Wistar rats. Food Chem Toxicol. 2010; 48(7): 1969-1972.

Page 13/20 
51. Teixeira M, Chaguri L do CA Guinski, Carissimi, AS, de Souza NL, Mori CMC, Gomes VMW, et al. Hematological and biochemical profiles of rats (Rattus norvegicus) kept under microenvironmental ventilation system. Braz. J Vet Res Anim Sci. 2000. 37(5): 341-347.

52. Giknis MLA, Clifford CB. Clinical laboratory parameters for Crl: Wi(Han) rats. Charles River Laboratories International: Wilmington, MA. $2008 ; 1-17$.

53. Boehm O, Zur B, Koch A, Tran N, Freyenhagen R, Hartmann M, et al. Clinical chemistry reference database for Wistar rats and C57/BL6 mice. Biol Chem. 2007; 388(5): 547-554.

54. Rigacci L, Alterini R, Bernabei PA, Ferrini PR, Agati G, Monici M. Multispectral Imaging Autofluorescence Microscopy for the Analysis of Lymph-Node Tissues. Photochem Photobiol. 2000; 71(6): 737-742.

55. Wizenty J, Ashraf MI, Rohwer N, Stockmann M, Weiss S, Biebl M, et al. Autofluorescence: A potential pitfall in immunofluorescence - based inflammation grading. J immunologival Methods, 2018. 456: 28-37.

56. Koshani R, Madadlou A. A viewpoint on the gastrointestinal fate of cellulose nanocrystals. Trends Food Sci Technol. 2018. 71: p. 268-273.

57. Mackie A, Gourcy S, Rigby N, Moffat J, Capron I, Bajka B. The fate of cellulose nanocrystal stabilised emulsions after simulated gastrointestinal digestion and exposure to intestinal mucosa. Nanoscale, 2019. 11.

58. Martins D, Domingos de Carvalho F, Gama FM, Dourado F. Dry Bacterial cellulose and Carboxymethyl Cellulose formulations with interfacial-active performance: processing conditions and redispersion. Cellulose. 2020; 27(11): 6505-6520.

59. Walters MP, Kelleher J, Findlay JM, Srinivasan ST. Preparation and characterization of a [14C]cellulose suitable for human metabolic studies. Br J Nutr. 1989; 62(1): 121-129.

60. Tuason DC, Krawczyk GR, Bulliga G. Microcrystalline Cellulose, in Food Stabilisers, Thickeners and Gelling Agents, A. Imeson, Editor. Wiley-Blackwell: United Kingdom; 2009. p. 218-236.

61. Wüstenberg T. Microcrystalline Cellulose, in Cellulose and Cellulose Derivatives in the Food Industry, T. Wüstenberg, Editor. Wiley-VCH Verlag GmbH \& Co. KGaA: Germany; 2015. p. 143-184.

62. Rodrigues AC, Fontão Al, Coelho A, Leal M, Soares da Silva FAG, Wan Y, Dourado F, Gama M. Response surface statistical optimization of bacterial nanocellulose fermentation in static culture using a low-cost medium. New Biotechnol. 2019; 49: 19-27.

63. Williams JM, Duckworth CA, Vowell K, Burkitt MD, Pritchard DM. Intestinal Preparation Techniques for Histological Analysis in the Mouse. Curr Protoc Mouse Biol. 2016; 6(2): 148-168.

64. Samulin E, Alswady-Hoff M, Ervik T K, Skare Ø, Ellingsen D G, Zienolddiny S. Cellulose nanocrystals modulate alveolar macrophage phenotype and phagocytic function. Biomaterials. 2019; 203: 31-42.

\section{Figures}




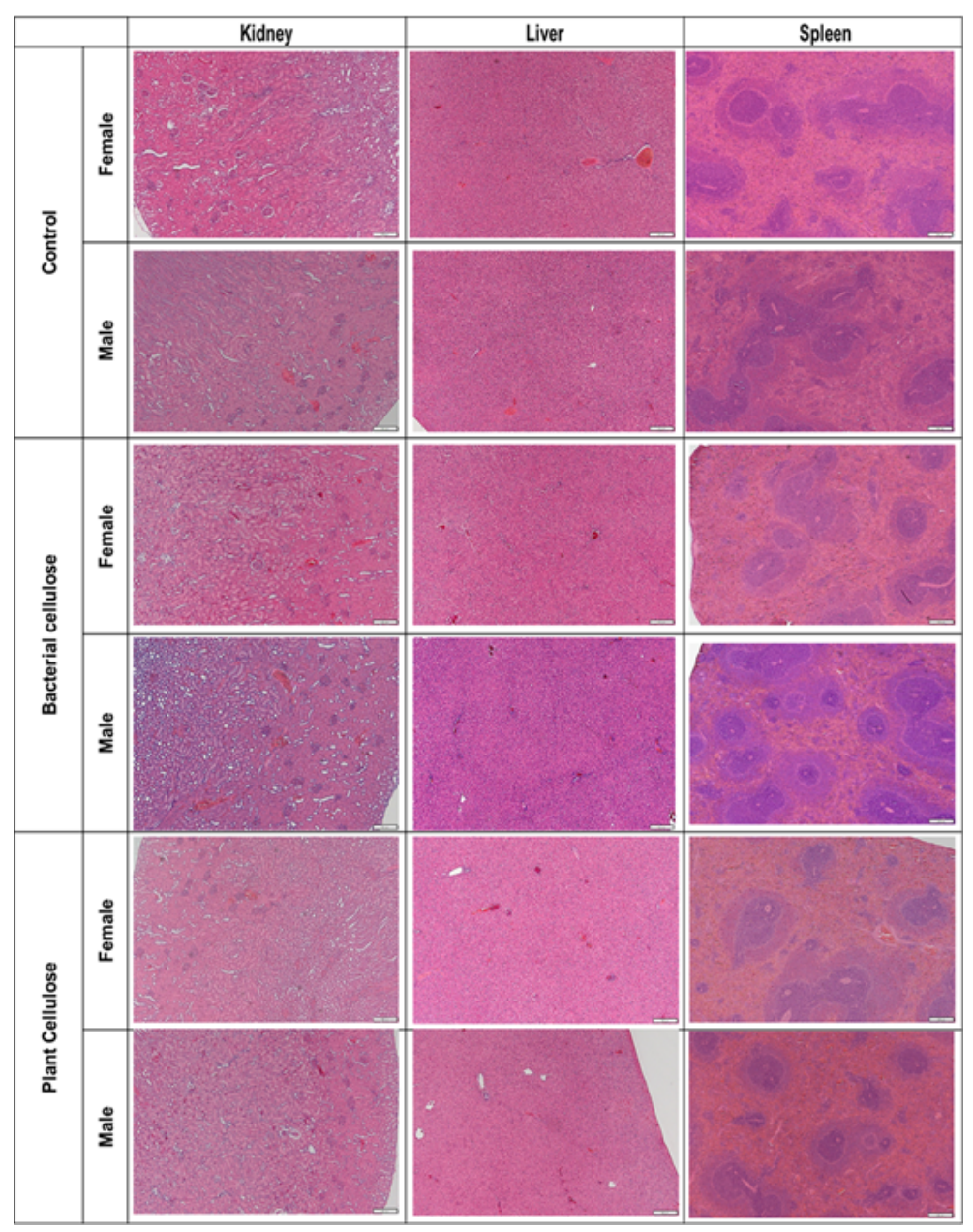

Figure 1

Representative photomicrographs of the histological sections of the collected organs (kidneys, liver and spleen) from all experimental groups stained with Hematoxylin and Eosin (H\&E). Scale bar $200 \mu \mathrm{m}$. 


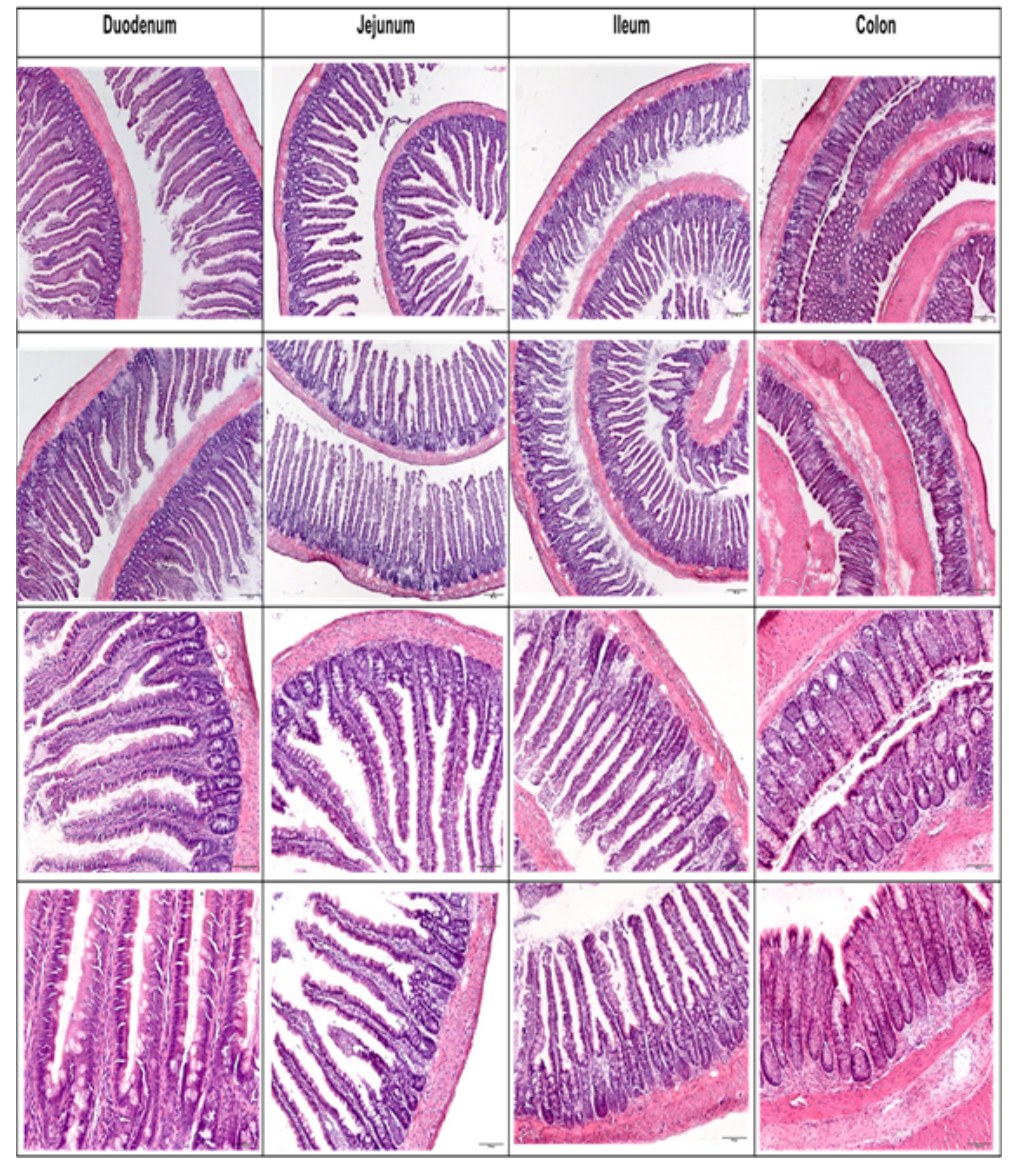

Figure 2

Representative H\&E photomicrographs of the histological sections of distinct regions of the intestine of female rats from the bacterial cellulose group. Scale bars- $200 \mu \mathrm{m}$ (line 1 and 2) and $100 \mu \mathrm{m}$ (line 3 and 4).

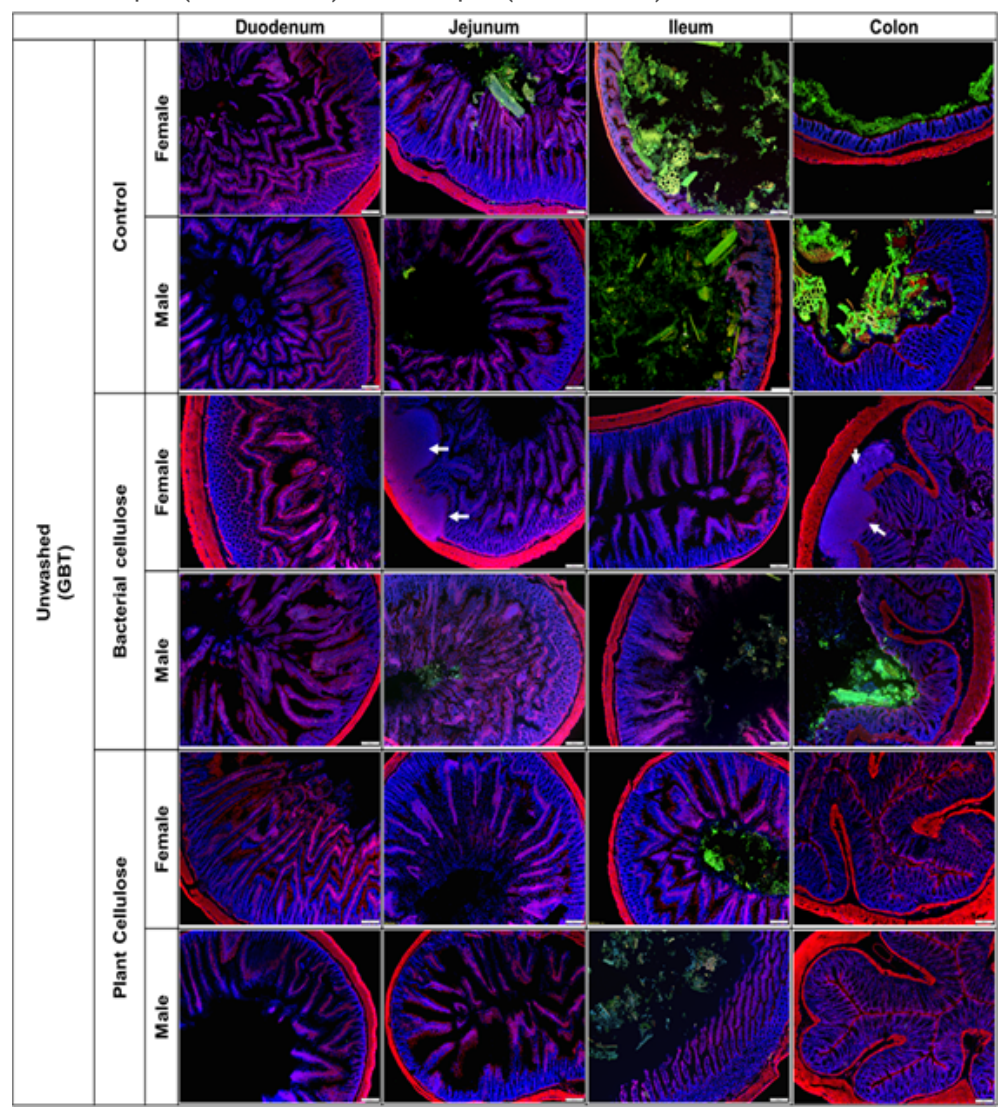




\section{Figure 3}

Representative fluorescence photomicrographs of histological sections of unwashed intestines processed by GBT, stained for cellulose (green), cytoskeleton (red) and nucleus (blue). Lymphoid tissues - peyer patches and colonic patches (highlighted by white arrows). Scale bar $200 \mu \mathrm{m}$.

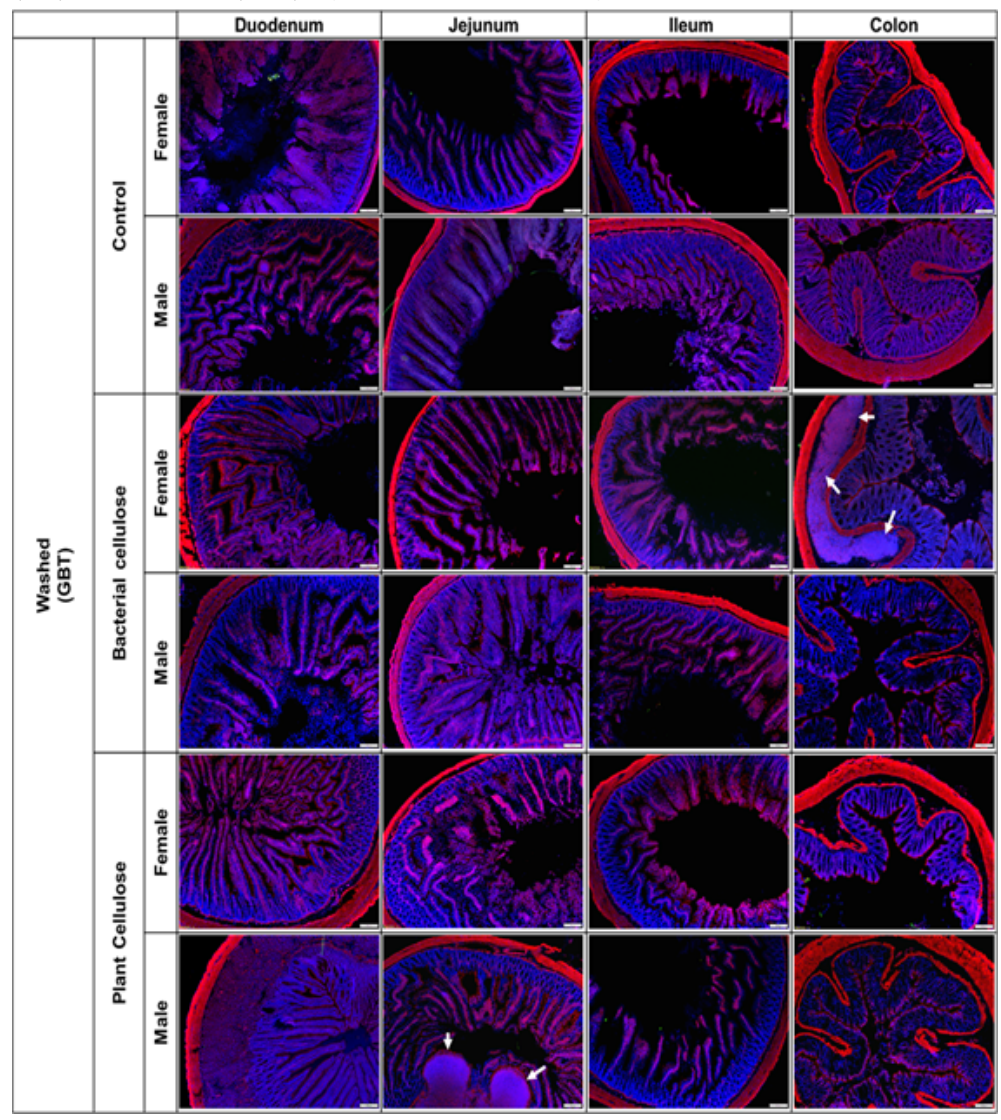

\section{Figure 4}

Representative fluorescence photomicrographs of histological sections of washed intestines processed by GBT, stained for cellulose (green), cytoskeleton (red) and for nucleus (blue) visualization. Lymphoid tissues - peyer patches and colonic patches (highlighted by white arrows). Scale bar $200 \mu \mathrm{m}$. 


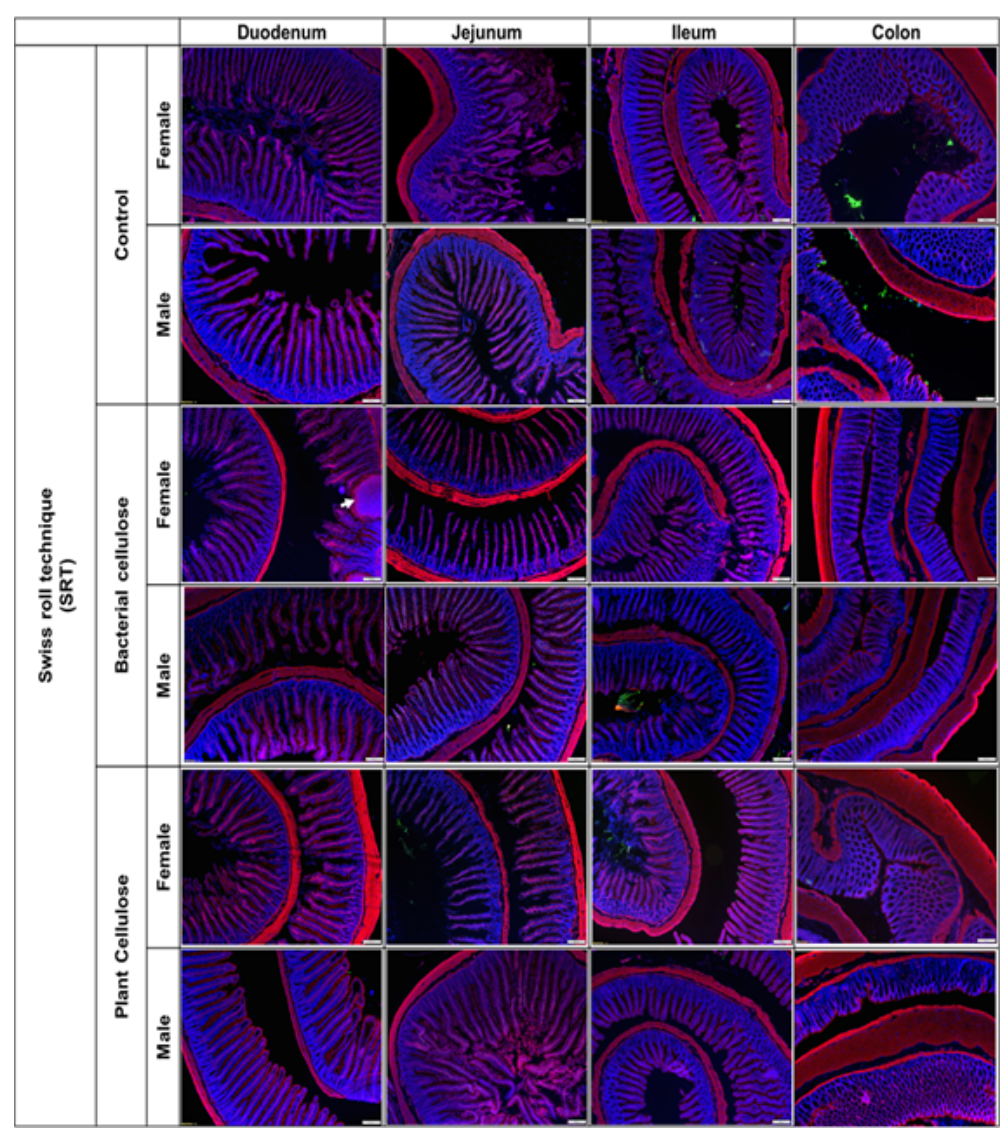

\section{Figure 5}

Representative Fluorescence photomicrographs of histological sections of washed intestines processed by SRT, stained for cellulose (green), cytoskeleton (red) and nucleus (blue). Lymphoid tissues - Peyer patches and colonic patches (highlighted by white arrows). Scale bar $200 \mu \mathrm{m}$. 


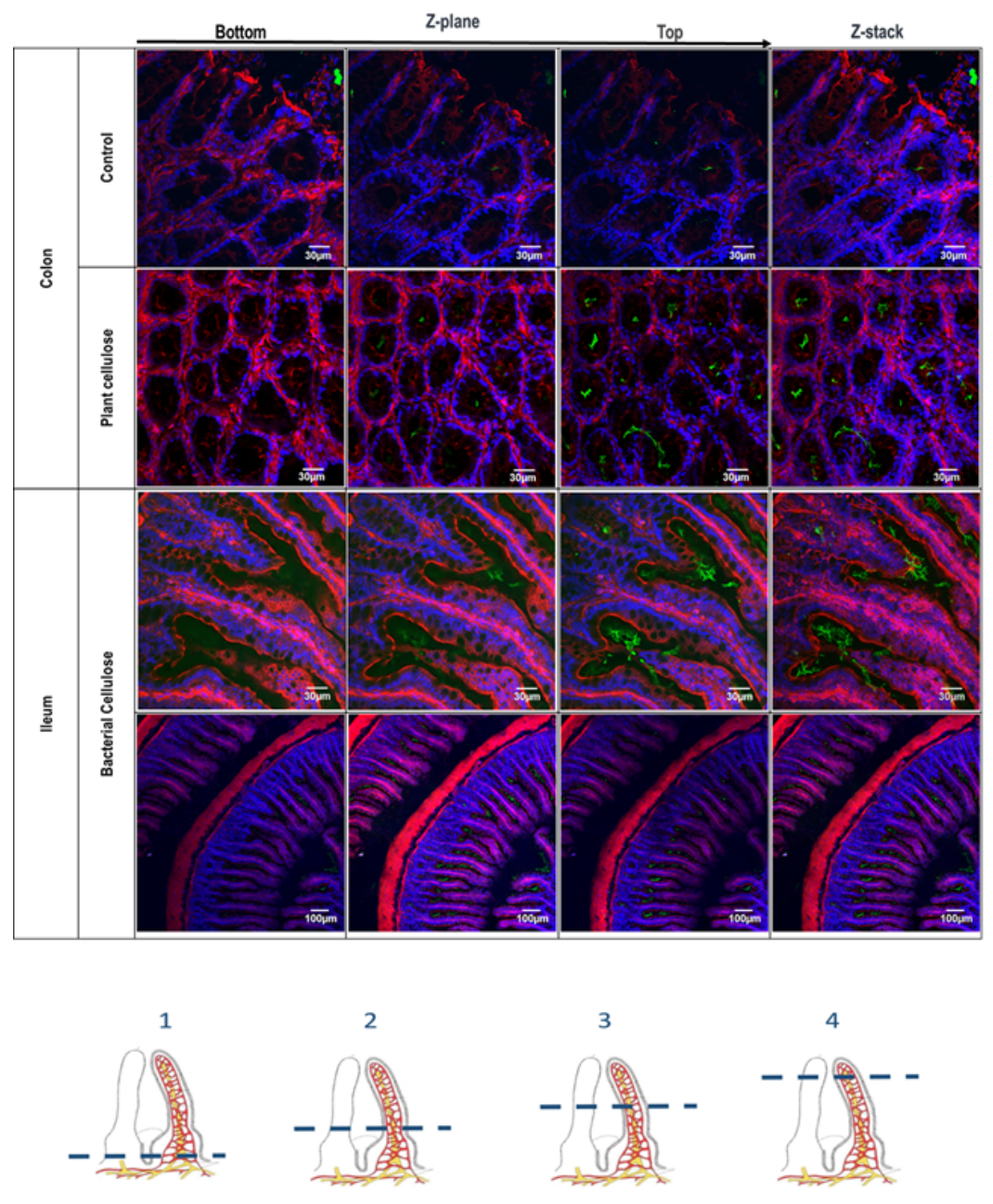

Figure 6

Different z-planes of confocal fluorescence photomicrographs of histological sections from the washed ileum and colon, processed by SRT and stained for cellulose (green), cytoskeleton (red) and nucleus (blue). From bottom to top (in each row, from left to right). Scale bars $-30 \mu \mathrm{m} ; 100 \mu \mathrm{m}$.

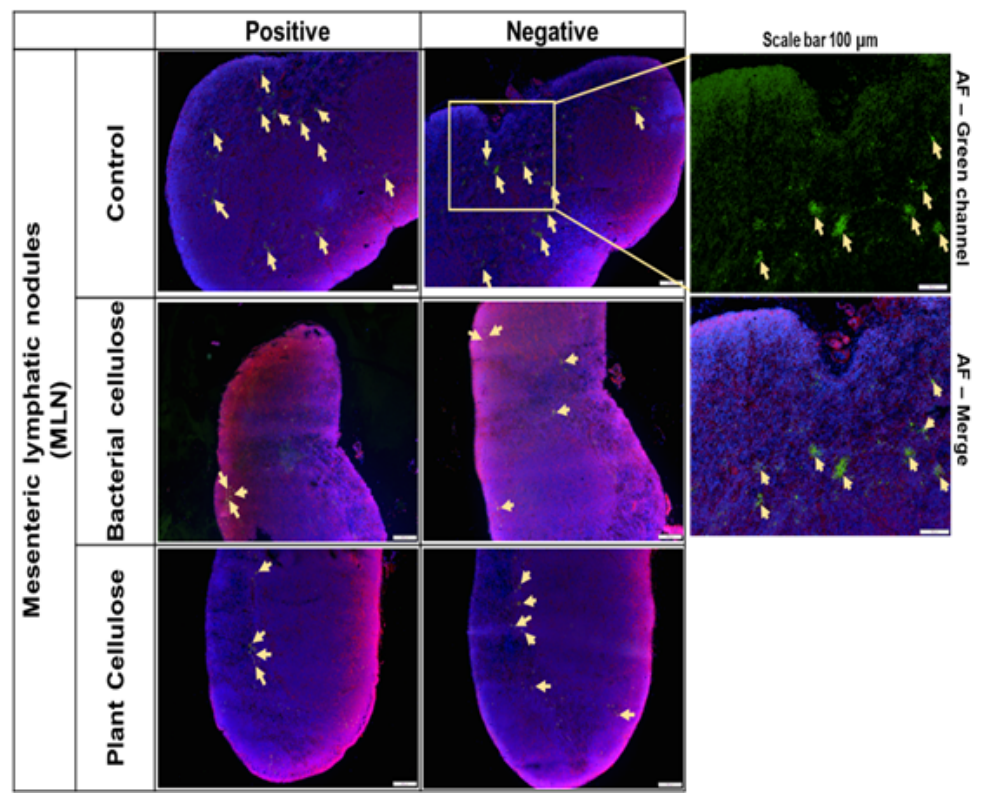

Figure 7 
Representative fluorescence photomicrographs of the mesenteric lymph nodes (MLN) stained for cellulose (green), cytoskeleton (red) and nucleus (blue) and the rounded diffuse green spots (yellow arrows) in the histological samples of the MLN of the different experimental groups, are visible in both positive and negative controls for cellulose staining (better observed in magnified image, on green channel and on corresponding merged one). Scale bar $200 \mu \mathrm{m}$.

\section{Supplementary Files}

This is a list of supplementary files associated with this preprint. Click to download.

- Onlinegraphicalabstracttiff.png 